NOUVELle

\section{Maladie de Huntington}

\section{Une neuro-dégénérescence qui prend sa source dans le développement cérébral ?}

Alexandra Durr ${ }^{1}$, Sandrine Humbert ${ }^{2}$

${ }^{1}$ Sorbonne Université, Institut du cerveau, AP-HP, Inserm, CNRS,

Hôpital Pitié-Salpêtrière,

47 boulevard de l'Hôpital,

75013 Paris, France.

${ }^{2}$ Univ. Grenoble Alpes, Inserm U1216,

Grenoble Institut Neurosciences, Chemin

Fortuné-Ferrini, 38700 La Tronche, France.

alexandra.durr@sorbonne-université.fr

sandrine.humbert@inserm.fr

> La maladie de Huntington est une maladie neurologique rare et héréditaire qui se manifeste généralement à l'âge adulte. Elle est causée par une expansion anormale de la répétition d'un triplet CAG dans la séquence nucléotidique $\mathrm{du}$ gène codant la huntingtine. Comme pour les autres maladies causées par une expansion similaire dans la séquence nucléotidique d'un gène, mais aussi comme pour d'autres maladies génétiques neurodégénératives se déclarant à l'âge adulte, incluant la sclérose latérale amyotrophique ou les maladies d'Alzheimer et de Parkinson dans leurs formes héréditaires, le gène dont la mutation est responsable de la maladie de Huntington est exprimé dès les premiers jours de la vie embryonnaire. Son expression est d'ailleurs indispensable pour le développement de l'embryon, comme en témoigne la mort in utero, à 7 jours et demi de développement, des souris invalidées pour ce gène [1]. La huntingtine intervient à plusieurs étapes du développement du cortex cérébral. L'étude d'un modèle murin de la maladie a montré que la huntingtine mutante perturbe le déroulement normal de ces étapes, ce qui conduit à une diminution de l'épaisseur corticale [2]. De plus, l'expression de la huntingtine mutante ou une diminution de la quantité de huntingtine normale pendant la période de développement cérébral suffit à produire des phénotypes caractéristiques de la maladie de Huntington chez les souris adultes $[3,4]$. L'ensemble de ces observations suggère qu'il pourrait exister une composante développementale dans la maladie de Huntington. $\varepsilon n$ accord avec cette hypothèse, des études de neuroimagerie réalisées chez des enfants porteurs de l'expansion anormale de la répétition du triplet CAG à l'origine de la maladie de Huntington ont révélé, dès l'âge de sept ans, c'est-à-dire bien avant l'apparition des premiers symptômes, l'existence d'un volume intracrânien plus faible que celui d'autres enfants du même âge [5]. Chez des individus adultes également porteurs de cette expansion anormale, l'imagerie par résonance magnétique (IRM), répétée à un an d'intervalle, a permis de constater une diminution du volume neuronal dans le cortex cérébral, précédant l'apparition des symptômes de plusieurs années [6]. Des neurones dérivés de cellules souches pluripotentes induites (IPS) provenant d'individus porteurs de l'expansion anormale présentent des changements dans l'expression de différents gènes impliqués dans le développement cérébral [7]. Enfin, la huntingtine mutante modifie l'identité neuronale dans les populations de cellules corticales d'organoïdes cérébraux [8]. Pour tenter de comprendre comment la huntingtine mutante affecte le développement cérébral humain, nous avons étudié le développement cérébral de fœtus humains porteurs d'une répétition anormale du triplet CAG à l'origine de la maladie de Huntington [9]. Les fragments de cerveau analysés proviennent de demandes d'interruption médicale de grossesse après un test génétique pré-symptomatique chez la mère ou le père et un test génétique prénatal chez le fœtus [10]. Nous avons obtenu, à la $11^{\mathrm{e}}$ semaine d'âge gesta- tionnel (13e semaine d'aménorrhée), des tissus intacts du cortex cérébral de quatre fœtus porteurs de la mutation qui les prédisposait à la survenue de la maladie après l'âge de 40 ans, et de quatre fœtus témoins.

Pourquoi s'intéresser au cortex cérébral ? Bien que le striatum soit une des premières structures à présenter une perte neuronale dans la maladie de Huntington, ce n'est pas la première à présenter un dysfonctionnement. En effet, les résultats de plusieurs études soutiennent l'hypothèse selon laquelle la dégénérescence du striatum dans la maladie de Huntington est secondaire à un dysfonctionnement du circuit cortico-striatal [1]. À 11 semaines de développement embryonnaire, les neurones corticaux générés sont ceux des couches profondes du cortex, qui projettent leurs axones vers le striatum et qui sont particulièrement affectés dans la maladie de Huntington à des stades tardifs de la maladie. Les neurones corticaux proviennent de la division des cellules progénitrices de la zone ventriculaire. Ces cellules envoient de fins prolongements vers les surfaces apicale et basale du neuroépithélium, et leur noyau se déplace le long de l'axe apicobasal de concert avec la progression du cycle cellulaire [11]. Ce processus de migration nucléaire intercinétique, commun au développement des neuroépithéliums pseudostratifiés, permet de maintenir l'équilibre entre le renouvellement et la différenciation des cellules progénitrices de la zone ventriculaire en contrôlant l'exposition de leurs noyaux à des signaux prolifératifs ou neurogènes. 


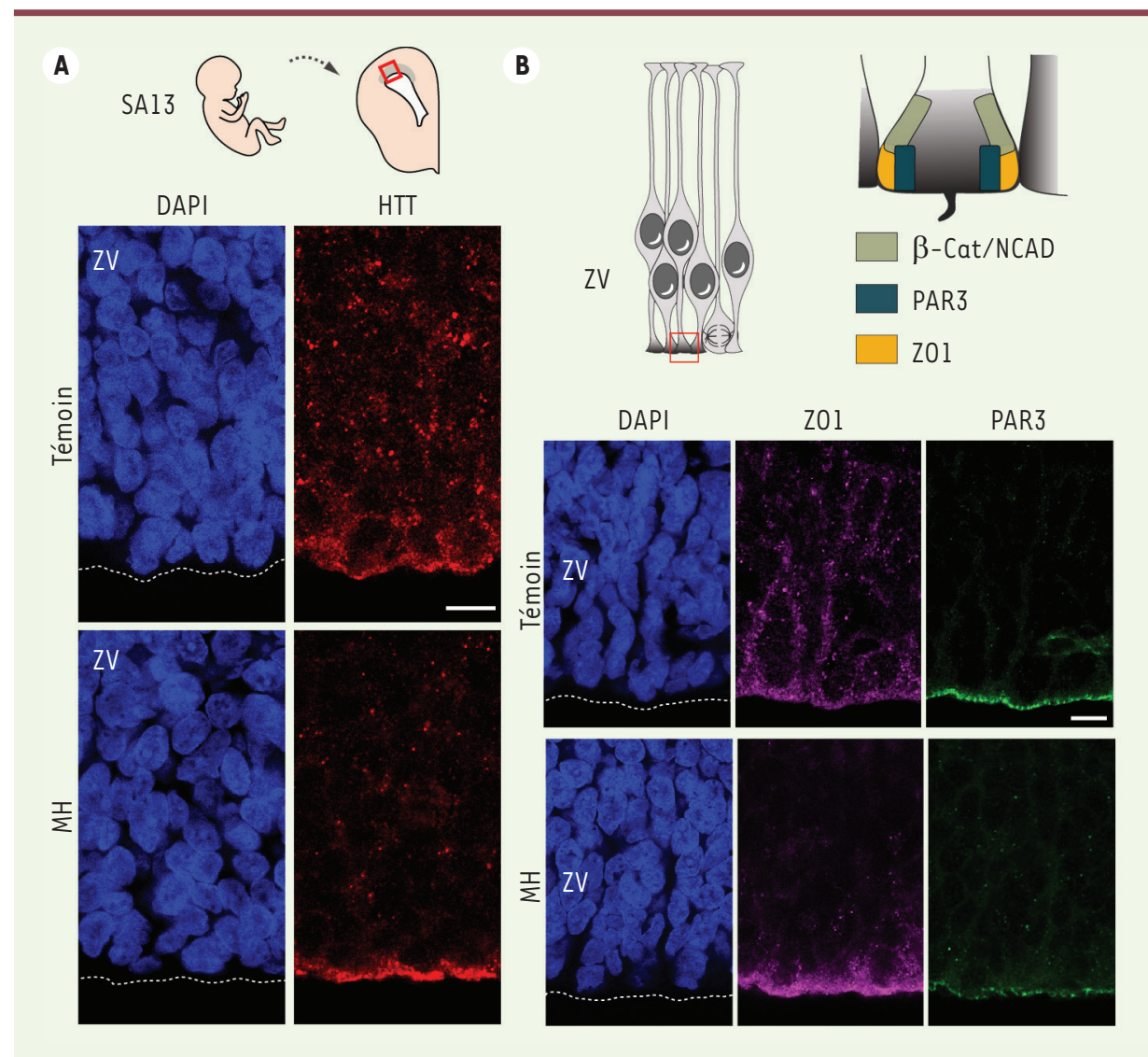

Figure 1. Dans la maladie de Huntington, l'intégrité du neuroépithélium du cortex cérébral est altérée dès la période de développement fœtal. A. Schéma montrant la zone ventriculaire (ZV) du cortex cérébral analysée (carré rouge) d'une part chez un fœtus témoin à 11 semaines d'âge gestationnel (soit 13 semaines d'aménorrhée, SAl3), et d'autre part chez un fœtus du même âge, porteur d'un nombre anormalement élevé de répétitions du triplet CAG dans la séquence nucléotidique du gène codant la huntingtine, et qui aurait été atteint de la maladie de Huntington à l'âge adulte (MH). Les coupes coronales de cerveau sont marquées à l'aide de trois anticorps spécifiques détectant respectivement la huntingtine (HTT) (A), la protéine de jonction intercellulaire $Z 01$ et la protéine de polarité cellulaire PAR-3 (B). Les noyaux cellulaires sont colorés en bleu par le 4',6-diamidino-2-phénylindole

(DAPI). La surface apicale du neuroépithélium est indiquée par un trait pointillé. B. Schéma montrant le prolongement apical des cellules neuroépithéliales progénitrices et leur cil primaire, ainsi que la distribution des protéines de jonctions intercellulaires Z01, N-cadhérine (NCAD) et $\beta$-caténine ( $\beta$-Cat). Barres d'échelle : $10 \mu \mathrm{m}$.

Nous avons d'abord analysé la distribution de la huntingtine qui, chez les fœtus témoins, délimite la surface apicale du neuroépithélium et est plus diffuse dans sa région basale. Chez les fœtus porteurs de la mutation, la huntingtine est anormalement concentrée à la surface apicale du neuroépithélium (Figure 1). Cette surface est constituée par les extrémités des prolongements apicaux des cellules neuroépithéliales progénitrices unis par des jonctions intercellulaires, elles-mêmes constituées par différentes protéines membranaires et sous-membranaires, telles que $\mathrm{ZOl}$ (zonula occludens-1), PAR-3 (partitioning defective 3 ), la $N$-cadhérine et la $\beta$-caténine. Or la huntingtine contrôle le trafic de ces protéines, qui est affecté par la huntingtine mutante [1]. Nous avons donc émis
I'hypothèse que la huntingtine mutante pourrait empêcher leur adressage correct aux jonctions intercellulaires. II existe une colocalisation de la huntingtine avec Z01, PAR-3, la N-cadhérine et la $\beta$-caténine dans la région apicale du neuroépithélium. Les niveaux d'expression de Z01, de la $\mathrm{N}$-cadhérine et de la $\beta$-caténine, qui sont élevés à la surface apicale du neuroépithélium dans la zone ventriculaire des fœtus témoins, le sont encore plus chez les fœtus porteurs de la mutation, avec une réduction concomitante de l'expression de ces protéines dans la région basale du neuroépithélium (Figure 1). Il existe également une anomalie de la distribution de PAR-3 chez les fœtus porteurs de la mutation: le niveau d'expression de cette protéine est diminué, de sorte que la démarcation de la surface apicale du neuroépithélium dans les échantillons tissulaires issus de ces fœtus et marqués par un anticorps spécifique de la protéine, est atténuée (et non pas intensifiée comme pour la $\mathrm{N}$-cadhérine, la $\beta$-caténine, et Z01).

Pour analyser plus précisément ces désorganisations et leurs conséquences, nous nous sommes tournées vers un modèle murin de la maladie de Huntington. Remarquablement, la huntingtine et les protéines des jonctions intercellulaires présentent les mêmes anomalies de distribution dans le neuroépithélium que chez les fœtus humains. Nous avons par ailleurs observé que déjà à ce stade précoce du développement, la huntingtine mutante empêche le trafic des endosomes, ce qui pourrait être à l'origine des défauts de distribution de 


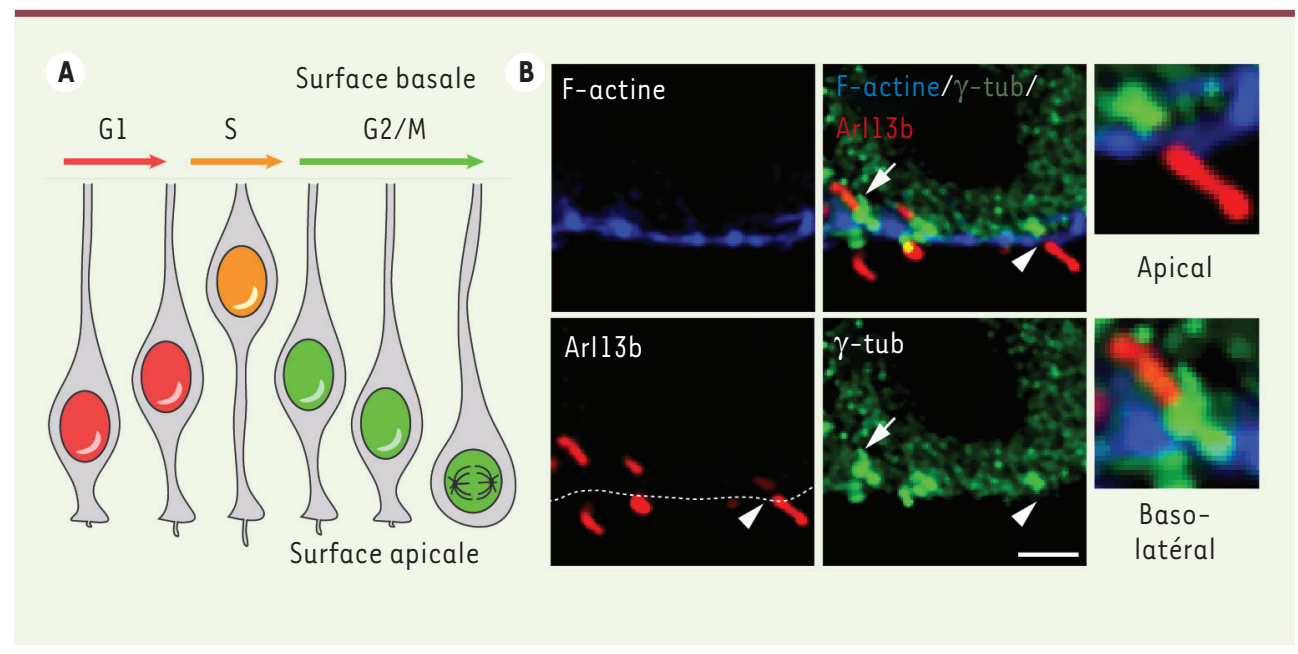

Figure 2. La migration nucléaire intercinétique et le cil primaire de la cellule neuroépithéliale. A. Schéma montrant le déplacement du noyau des cellules neuroépithéliales progénitrices entre les pôles apical et basal pendant la progression du cycle cellulaire. Gl, $S, G 2 / M$ : phase $G 1$, phase $S$, et transition G2-mitose. B. Une orientation baso-latérale des cils primaires est révélatrice de la production de cellules progénitrices basales, davantage

engagées dans le lignage neuronal que les cellules progénitrices dont la localisation est plus apicale. La flèche désigne un cil en position basolatérale, et la tête de flèche un cil en position apicale. Le marquage de la F-actine délimite la surface cellulaire apicale, tandis que celui de la $\gamma$-tubuline $(\gamma$-tub) indique la base du cil ; Arl13b est un marqueur spécifique du cil primaire. La surface apicale du neuroépithélium est indiquée par un trait pointillé. Barre d'échelle : $2 \mu \mathrm{m}$.

Z01, de PAR-3, de la N-cadhérine et de la $\beta$-caténine. Ce modèle murin a également permis d'étudier la migration nucléaire intercinétique, dont le bon déroulement nécessite l'intégrité des extrémités des prolongements apicaux des cellules neuroépithéliales (Figure IB). Pendant la phase Gl du cycle cellulaire, les noyaux des cellules progénitrices nées à la surface apicale du neuroépithélium se déplacent vers le pôle basal, où se déroule la phase de réplication de l'ADN (phase $S$ ), puis retournent pendant la phase $\mathrm{G} 2$ vers la surface apicale, où les cellules se divisent (phase M) (Figure 2A). Pour mesurer les déplacements du noyau cellulaire vers les surfaces basale (phase $\mathrm{Gl}$ ) et apicale (phase G2) in vivo chez la souris, nous avons utilisé l'expression de protéines fluorescentes révélant les différentes phases du cycle cellulaire. Chez les embryons de souris modèles de la maladie de Huntington, les noyaux en migration se déplacent plus lentement durant les phases $G 1$ et $G 2$ que ceux des embryons témoins, ce qui entraîne un allongement de ces phases, alors que la transition Gl/S est raccourcie. Nous avons également trouvé un indice mitotique plus faible chez les embryons de souris modèles de la maladie de Hun- tington que chez les embryons témoins, et avons retrouvé cette différence chez les fœtus humains que nous avons analysés [9].

Les cellules du neuroépithélium possèdent à leur surface apicale un cil primaire, dont l'assemblage et le désassemblage suit la progression du cycle (Figure 2). Le marquage par un anticorps spécifique a révélé que la longueur et la densité des cils dans la région apicale du neuroépithélium étaient augmentées dans les échantillons issus des fœtus murins et humains porteurs de la mutation, ce qui confirme que les cellules ne progressent pas correctement dans le cycle cellulaire. Comme une phase Gl plus longue et une transition $\mathrm{Gl} / \mathrm{S}$ plus courte caractérisent les cellules progénitrices engagées vers le lignage neuronal, nous avons émis l'hypothèse que la huntingtine mutante pourrait favoriser la production de cellules progénitrices basales, qui sont davantage engagées dans la différenciation neuronale que les cellules progénitrices situées dans la région apicale du neuroépithélium [11]. En effet, dans les échantillons murins et humains porteurs du gène muté à l'origine de la maladie de Huntington, nous avons observé une plus grande proportion de cellules progénitrices basales. Une analyse de la disposition des cils primaires, dont l'orientation baso-latérale est révélatrice de la production de cellules progénitrices basales, nous a permis de confirmer cette observation (Figure 2).

$\varepsilon$ n résumé, dans la zone ventriculaire du cerveau des fœtus humains porteurs d'une mutation responsable de la maladie de Huntington, la huntingtine mutante et certaines protéines impliquées dans les jonctions intercellulaires sont anormalement distribuées et la polarité du neuroépithélium est modifiée. Le cycle cellulaire des cellules apicales est perturbé, entraînant une diminution du nombre des cellules neuroépithéliales en train de proliférer et une augmentation du nombre des progéniteurs neuronaux entrant prématurément dans la spécification du lignage neuronal. Ces résultats indiquent que la huntingtine est impliquée dans le contrôle de l'équilibre entre prolifération et différenciation cellulaires au cours du développement. Les défauts du développement cérébral que nous avons observés pourraient rendre ultérieurement le circuit neuronal cortico-striatal plus vulnérable aux dysfonctionnements qui caractérisent la maladie de Huntington. Ainsi, lorsqu'un traitement sera 
disponible, il devra probablement être administré tôt, bien avant le début des symptômes. Il reste à comprendre en quoi ces défauts précoces du développement contribuent au dysfonctionnement cérébral et à la dégénérescence neuronale qui suivent à l'âge adulte, et comment leur compensation se met très tôt en place et se maintient pendant la longue période silencieuse sans symptômes cliniques (qui dure généralement plusieurs décennies). D'autres études chez l'homme et dans des modèles animaux de la maladie seront nécessaires pour tenter de répondre à ces questions. $\diamond$

\section{Huntington disease: Neurodegeneration rooted in brain development?}

\section{REMERCIEMENTS}

Les auteures remercient les personnes concernées par la maladie de Huntington pour leur aide inconditionnelle à la recherche sur cette maladie, ainsi que
M. Barnat pour certaines illustrations de cet article. Ce travail a été cofinancé par l'Agence nationale pour la recherche, la Fondation pour la recherche médicale et la Fondation pour la recherche sur le cerveau.

\section{LIENS D'INTÉRÊT}

Les auteures déclarent n'avoir aucun lien d'intérêt concernant les données publiées dans cet article.

\section{RÉFÉRENCES}

1. Saudou F, Humbert S. The biology of huntingtin. Neuron $2016 ; 89: 910-26$

2. Molina-Calavita M, Barnat M, Elias S, et al. Mutant huntingtin affects cortical progenitor cell division and development of the mouse neocortex. J Neurosci 2014 ; 34 : 10034-40.

3. Arteaga-Bracho દE, Gulinello M, Winchester ML, et al. Postnatal and adult consequences of loss of huntingtin during development: Implications for Huntington's disease. Neurobiol Dis 2016 ; 96 : 144-55.

4. Molero AE, Arteaga-Bracho $\varepsilon \varepsilon$, Chen $\mathrm{CH}$, et al. Selective expression of mutant huntingtin during development recapitulates characteristic features of Huntington's disease. Proc Natl Acad Sci USA 2016 ; $116: 5736-41$.
5. Lee JK, Mathews K, Schlaggar B, et al. Measures of growth in children at risk for Huntington disease. Neurology $2012 ; 79$ : 668-74.

6. Tabrizi SJ, Langbehn DR, Leavitt BR, et al. Biologica and clinical manifestations of Huntington's disease in the longitudinal TRACK-HD study: cross-sectional analysis of baseline data. Lancet Neurol 2009 ; 8 : 791-801.

7. Consortium HDi. Developmental alterations in Huntington's disease neural cells and pharmacological rescue in cells and mice. Nat Neurosci $2017 ; 20: 648-60$

8. Conforti P, Besusso D, Bocchi VD, et al. Faulty neuronal determination and cell polarization are reverted by modulating HD early phenotypes. Proc Natl Acad Sci USA 2018 ; 115 : ع762-71.

9. Barnat M, Capizzi M, Aparicio $\varepsilon$, et al. Huntington disease alters human neurodevelopment. Science $2020 ; 369: 787-93$.

10. Bouchghoul H, Clement SF, Vauthier D, et al. Prenatal testing in Huntington disease: after the test, choices recommence. Eur J Hum Genet $2016 ; 24: 1535-40$.

11. Arai Y, Taverna $\varepsilon$. Neural progenitor cell polarity and cortical development. Front Cell Neurosci 2017 ; 11 : 384.

P ossédées du Malin au Moyen-Âge, les sorcières hystériques sont vouées au bûcher. Enfermées au xvII siècle, maltraitées, elles rejoignent la Cour des Miracles de l'Hospice de la Vieillesse-Femmes à la Salpêtrière... Jusqu'à ce que le Dr JeanMartin Charcot (1825-1893) mène le combat qui transforme l'ancien hospice en hôpital : l'École de la Salpêtrière de

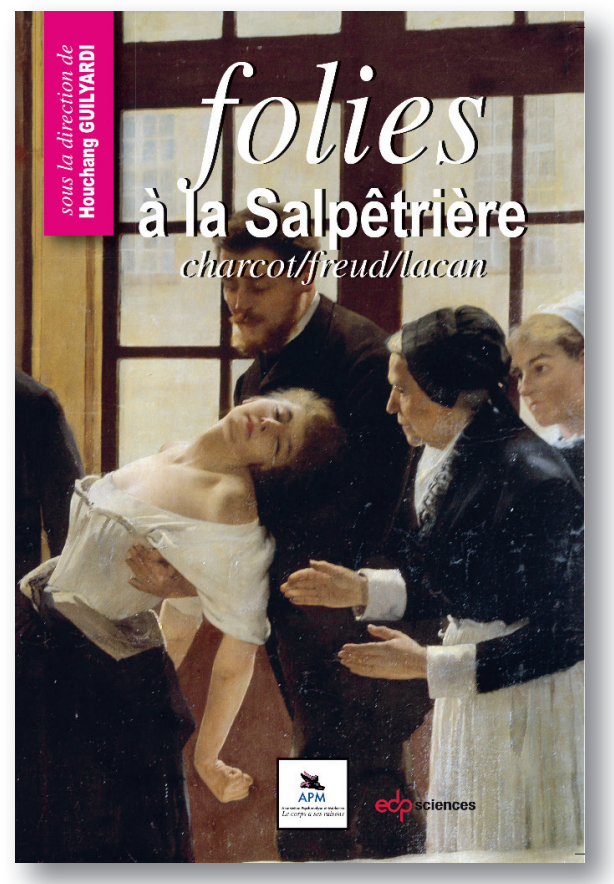

ISBN : $978-2-7598-1268-4$
240 pages

$20 €$
Paris est née, qui devient lieu de recherche, d'enseignement et de soins, de renommée internationale.

Jean Martin Charcot n'a pas bonne presse, et pourtant... Hystérie et folie traversent les siècles, prenant les formes de «l'air du temps ».

De l'utérus migrateur d'Hippocrate aux recherches neurologiques de Charcot. Du désir inconscient avec Freud à la jouissance du parlêtre chez Lacan... C'est à cette traversée historique et conceptuelle que nous convie cet ouvrage.

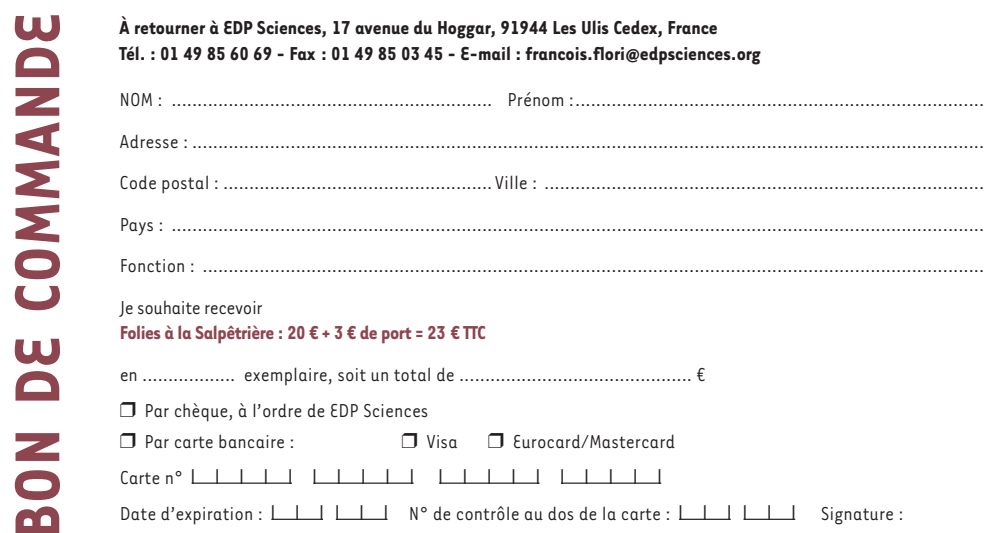

